\title{
AN ACTIVE, TRACKING MICROWAVE NOTCH FILTER USING A PAIR OF GUNN OSCILLATORS
}

\author{
Santosh Kumar Dawn and Taraprasad Chattopadhyay \\ ${ }^{1}$ Dept. of Physics, Visva-Bharati, Santiniketan, West-Bengal, India.
}

\begin{abstract}
:
In this paper, we have designed a signal tracking microwave notch filter at $\mathrm{X}$-band ( $8 \mathrm{GHz}-12.4 \mathrm{GHz})$ which is tunable. The experimental notch frequency is $9.42 \mathrm{GHz}$ and a 3-dB bandwidth of this filter is 105 $\mathrm{MHz}$. The theoretical response agrees well with the experimental response of this notch filter. The frequency can be tuned by tuning the Gunn oscillators. The Gunn oscillators being injection-locked to the input signal make the notch filter tracking in character.
\end{abstract}

\section{Keywords:}

Notch Filter, Microwaves, X-band, Gunn oscillators, Lockband, Active device.

\section{INTRODUCTION}

Microwave notch filters [1-19] are essential components of a microwave communication system. It finds application in satellite communication in order to eliminate interference which can produce jamming in a microwave receiver. It also finds application in microwave distortion analyzer where the notch filter suppresses the fundamental component. Another important application of the notch filter in $\mathrm{rf} /$ microwave domain is to separate the luminance signal of a colour television from the composite luminance-chrominance TV signals.

The microwave notch filter can be designed electronically [1-11] as well as optically [12-15]. Optical realizations of tunable microwave notch filter have reported in literature [12-15]. In this paper, we report the electronic design of a microwave notch filter which is tunable, active and signal tracking in character simultaneously. Notch filters are special cases of band reject filters [20-24] where the attenuation at a particular frequency becomes maximum and have a narrow bandwidth.

Looking into literature, it is seen that the microwave bandstop filters have been designed with barium strontium titanate thin film varactor technology [1]. A tunable narrowband microwave notch filter has been reported [2] to be designed at X-band with transmission minimum at $30 \mathrm{~dB}$ and bandwidth of $20 \mathrm{MHz}$. Jackwoski et.al. have designed [3,4] microwave tunable notch filter using microstrip configuration. Microwave filter design has been discussed in detail in [5-8]. Active microwave filters have been reported to be designed [9] using negative resistance devices. Simulation work on the design of microwave notch filter can be found in [10]. In a recent work, the authors have designed a microwave notch filter [11] using a pair of unilaterally injectionlocked Gunn oscillators. In the present design, the authors have designed a microwave active notch filter in which the injection signals to two Gunn oscillators are not in phase but they differ in phase which is signal frequency dependent. In the present filter, the locking signals injected 
into two Gunn oscillators differ in phase by $\frac{\pi}{2}$ radian. As a result, the mechanism of interference suppression is different in the present design from that reported in [11]. The experimental frequency response of the present notch filter is seen to fit with the theoretical response much better than that reported in [11].

\section{SYSTEM DESCRIPTION}

The circuit diagram of the microwave notch filter is shown in Fig.1.

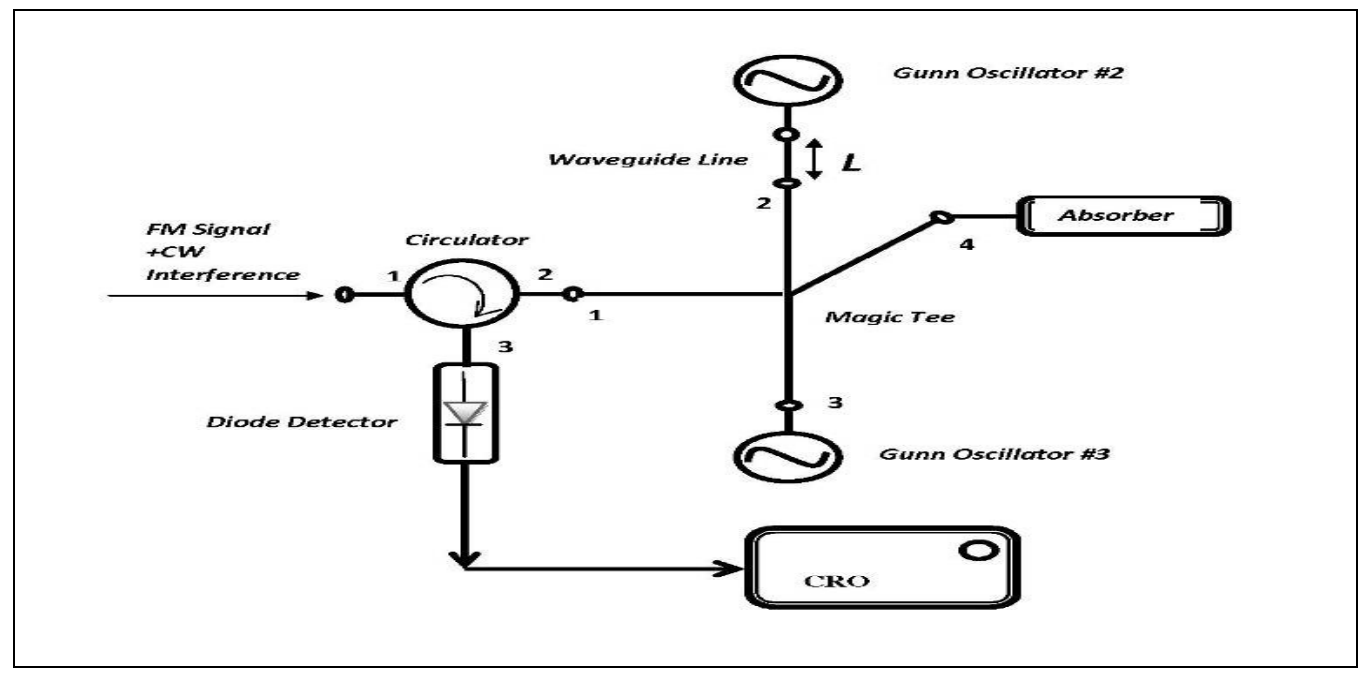

Fig. 1. Schematic diagram of the active, tracking microwave notch filter.

The notch filter is implemented using a magic tee, two identical Gunn oscillators, a waveguide section of length $L$, a circulator and a waveguide vane microwave absorber. The FM signal accompanied by $\mathrm{CW}$ interference enters port- $1(\mathrm{H}$-arm) of the magic tee and undergo half power division at the magic tee junction. One of these waves is injected into Gunn oscillator \#3 connected at the end of collinear arm-3 of the magic tee and makes oscillator fall in synchronism with the interfering tone which is to be eliminated. The other wave travels down arm-2 of the magic tee and propagates through the waveguide section of length ' $L$ ' which finally gets injected into Gunn oscillator \#2. The Gunn oscillator \#2 gets injection locked to the interfering tone. The Gunn oscillators \#2 and \#3 are identical. The composite microwave signal finally emerges through port- 1 of the magic tee. The input signal and the emerging output signal are separated by the circulator. The power of the emerging signal is measured by detecting it in a Schottky diode detector. The dc voltage output of the detector gives the measure of the microwave power input to the detector. This detector does not form any part of the notch filter. It is only used to measure the microwave power output.

\section{ANALYSIS}

The input signal is assumed to be FM. We perform a static analysis of the system. The input frequency is assumed to vary slowly. Let the microwave injection signal to Gunn Oscillator \#2 be represented by the voltage equation

$$
v_{i n j}(t)=V_{i} \sin \left(\omega_{i n} t-\theta_{L}\right)
$$


where $V_{i}$ is the voltage amplitude, $\omega_{i n}$ is the input signal frequency in radian and $\theta_{L}$ is the phase shift of the injection signal during its propagation through the waveguide of length " $L$ ". Here,

$$
\theta_{L}=\frac{\omega_{i n} L}{v_{p}\left(\omega_{i n}\right)}=\frac{L}{c} \sqrt{\omega_{i n}^{2}-\omega_{c o}^{2}}
$$

where $v_{p}\left(\omega_{i n}\right)$ is the phase velocity of the signal of radian frequency $\omega_{i n}$ and ' $c$ ' is the vacuum velocity of light. $\omega_{c o}$ is the cut-off frequency of the waveguide line. For Gunn oscillator \#3 connected with the port- 3 of the magic tee, $L=0 . \therefore \theta_{L}=0$.

The output of the locked oscillators are assumed to be of the form

$$
v_{o n}(t)=V_{o n} \sin \left(\omega_{i n} t-\phi_{n}\right)
$$

for $n=2,3 . V_{o n}$ is the voltage amplitude of oscillator $n($ for $n=2,3)$. $\phi_{n}$ is the output phase of the corresponding oscillator. For Gunn oscillator \#2, the input-output phase error is $\left(\phi_{2}-\theta_{L}\right)$ while the same for Gunn Oscillator \#3 is $\phi_{3}$. The phase equations for Gunn oscillator \#2 and \#3 are given by

$$
\frac{d \phi_{2}}{d t}=\frac{\omega_{02}}{2}\left(\frac{\omega_{i n}}{\omega_{02}}-\frac{\omega_{02}}{\omega_{i n}}\right)-\frac{\omega_{02}}{2 Q_{L}} \sqrt{\frac{P_{i n j}}{P_{02}}} \sin \left(\phi_{2}-\theta_{L}\right)
$$

and $\quad \frac{d \phi_{3}}{d t}=\frac{\omega_{03}}{2}\left(\frac{\omega_{i n}}{\omega_{03}}-\frac{\omega_{03}}{\omega_{i n}}\right)-\frac{\omega_{03}}{2 Q_{L}} \sqrt{\frac{P_{i n j}}{P_{03}}} \sin \phi_{3}$

respectively.

The free-running radian frequencies of Gunn oscillator \#2 and Gunn oscillator \#3 are $\omega_{02}$ and $\omega_{03}$ respectively. In experiment, $\omega_{02}$ and $\omega_{03}$ are made identical. $Q_{L}$ is the external Q-factor of the Gunn oscillators. $P_{i n j}$ is the injection power to Gunn oscillators which is the same for two oscillators. $P_{02}$ and $P_{03}$ are free running output powers of Gunn oscillators \#2 and \#3 respectively.

The detector input voltage, using complex representation, is given by

$$
v_{\text {Din }}(t)=\frac{1}{\sqrt{2}}\left(v_{02} e^{-j \theta_{L}}+v_{03}\right)
$$

Now,

$$
\left|v_{\text {Din }}(t)\right|^{2}=\frac{1}{2}\left(v_{02}^{2}+v_{03}^{2}+2 v_{02} v_{03} \cos \left(\phi_{2}-\phi_{3}-\theta_{L}\right)\right)
$$

In the steady state, $\frac{d \phi_{2}}{d t}=0$ and $\frac{d \phi_{3}}{d t}=0$

From equation (4), in the steady state, we get

$$
\phi_{2}-\theta_{L}=\sin ^{-1}\left[\frac{\omega_{i n}-\omega_{02}}{\Delta \omega_{H}}\right]
$$

where $\Delta \omega_{H}$ is the half lockband of the Gunn oscillator \#2. 
From (5), in the steady state, we get

$$
\phi_{3}=\sin ^{-1}\left[\frac{\omega_{i n}-\omega_{03}}{\Delta \omega_{H}}\right]
$$

We take $\omega_{02}=\omega_{03}=\omega_{0}$ (say). Equation (7) can now be recast as

$$
\left|v_{\text {Din }}(t)\right|^{2}=\frac{1}{2}\left[v_{02}^{2}+v_{03}^{2}+2 v_{02} v_{03} \cos \left\{\sin ^{-1}\left(\frac{\omega_{i n}-\omega_{0}}{\Delta \omega_{H}}\right)-\sin ^{-1}\left(\frac{\omega_{i n}-\omega_{0}}{\Delta \omega_{H}}\right)-2 \theta_{L}\right\}\right]
$$

The detector output voltage is given by

$$
v_{D o}=\eta \mid v_{\text {Din }}(t)^{2}=\frac{\eta}{2}\left[v_{02}^{2}+v_{03}^{2}+2 v_{02} v_{03} \cos \left\{\sin ^{-1}\left(\frac{\omega_{i n}-\omega_{0}}{\Delta \omega_{H}}\right)-\sin ^{-1}\left(\frac{\omega_{i n}-\omega_{0}}{\Delta \omega_{H}}\right)-2 \theta_{L}\right\}\right]
$$

The output phase $\phi_{2}$ and $\phi_{3}$ of the injection locked Gunn oscillator \#2 and Gunn oscillator \#3 as functions of input signal frequency are plotted in fig. 2 and fig. 3 respectively.

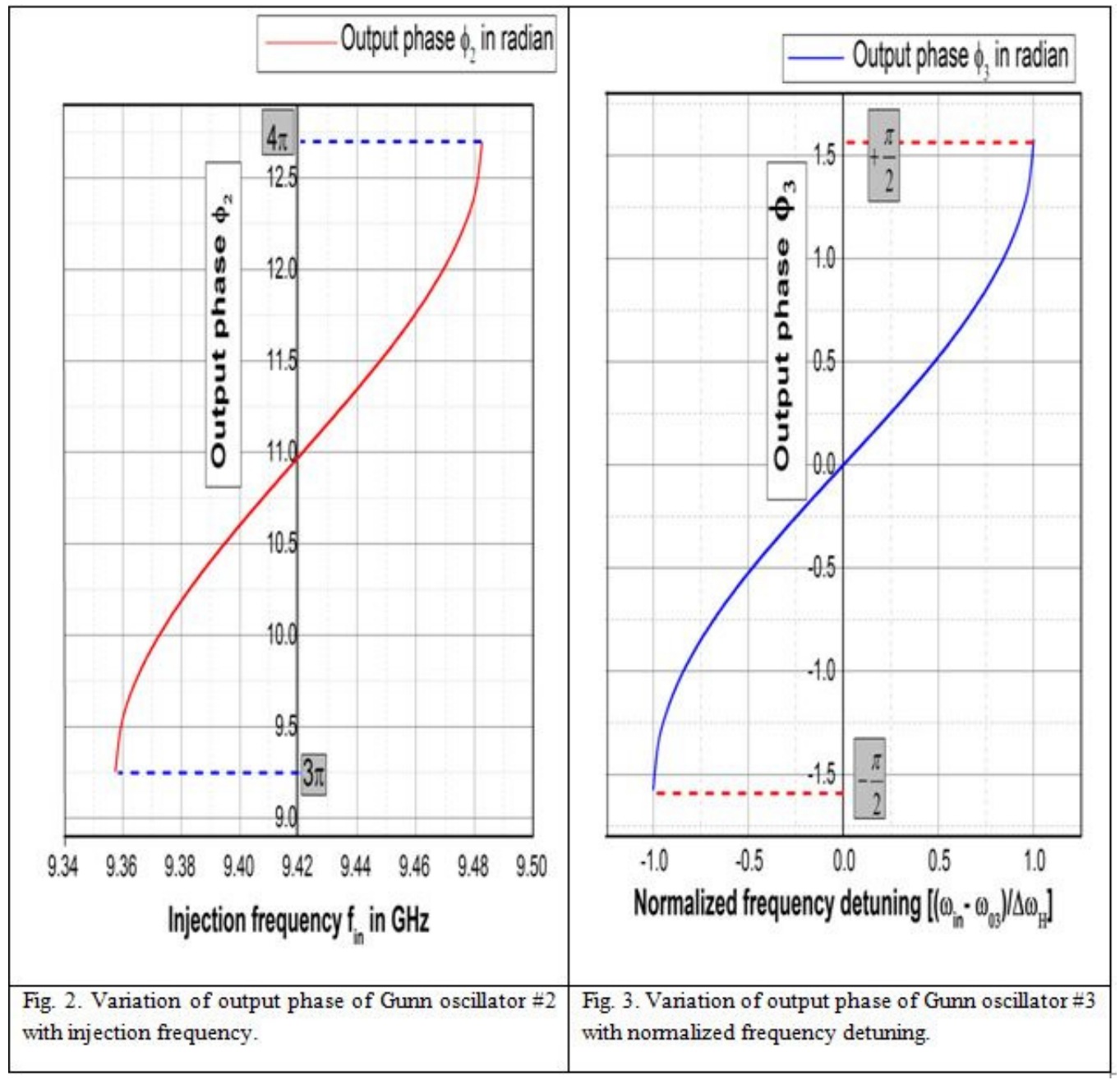


The input-output phase error, $\phi_{3}$, varies between $-\frac{\pi}{2}$ and $+\frac{\pi}{2}$ radian when the input signal frequency is swept from the lower end of the lockband to the upper end of the lockband. The output phase $\phi_{2}$ of the Gunn oscillator \#2 varies from $3 \pi$ radian to $4 \pi$ radian when the input signal frequency is swept from the lower end of the lockband to the upper end of the lockband. The waveguide line of length " $L$ " inserted between the port- 2 of the magic tee and Gunn oscillators \#2 produces a phase shift of the injection signal by an amount $\theta_{L}=\frac{2 \pi L}{c} \sqrt{f_{i n}^{2}-f_{c o}^{2}}$. At the notch frequency $f_{n}=9.42 \mathrm{GHz}, \theta_{L}$ is calculated to be $\frac{7 \pi}{2}$ radian. Thus, at the notch frequency the locking signals for Gunn oscillators \#2 and Gunn oscillators \#3 differ in phase by $\frac{7 \pi}{2}$ radian. The experimental response of the notch filter is shown in fig. 4 .

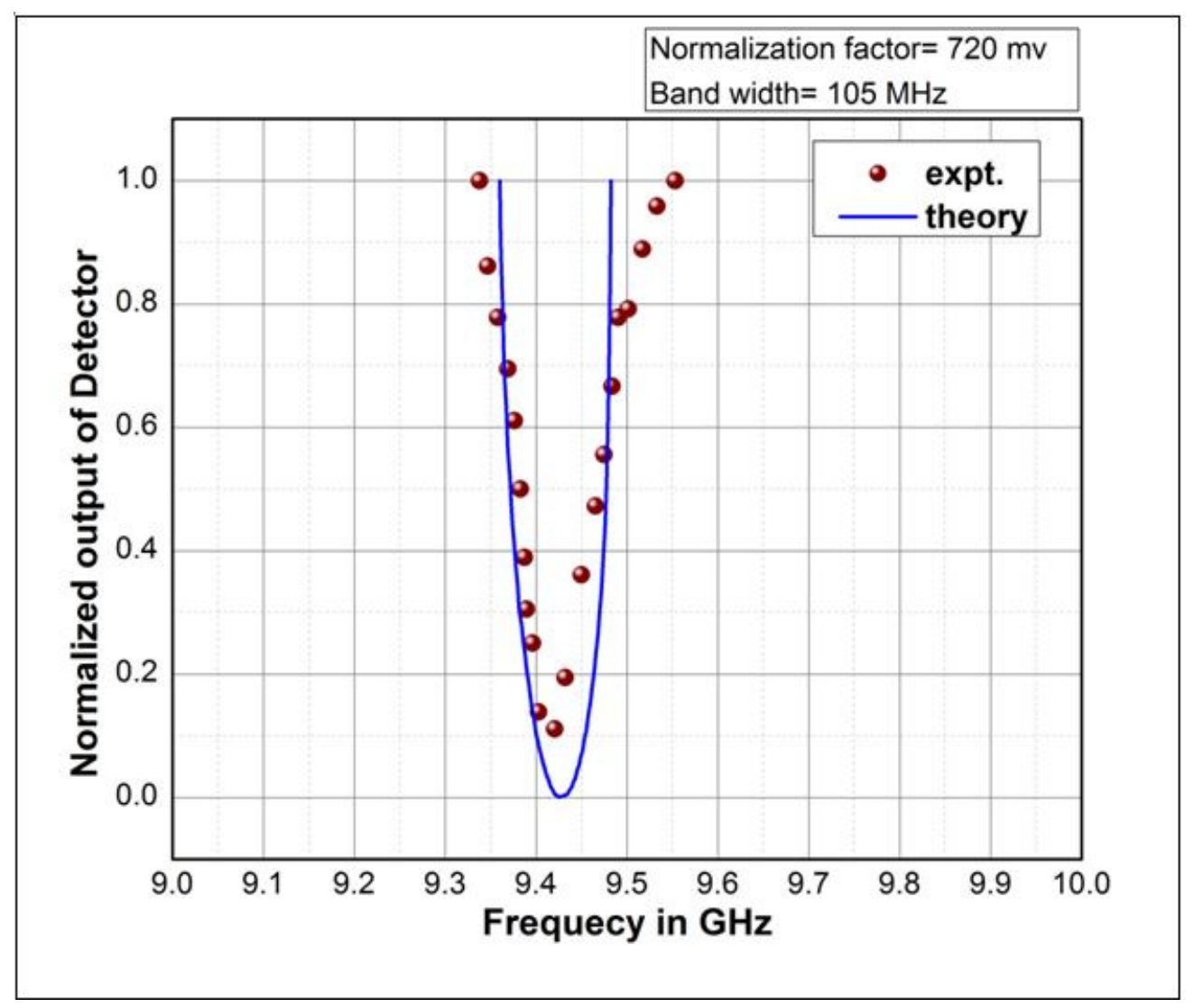

Fig. 4. Theoretical and experimental frequency response of the proposed notch filter

The 3-dB bandwidth of the notch filter measured experimentally is $105 \mathrm{MHz}$. The calculated response of the notch filter fits well with the experimental response. The Gunn oscillators are micrometer-tunable. So, the notch frequency can also be tuned by tuning the Gunn oscillators. The input signal tracking character of the designed microwave notch filter originates from the injection locking technique being employed in the design. The microwave diode detector is not any part of the notch filter. It is only used to measure the output microwave power by detecting the microwave signal appearing at its input. 


\section{CONCLUSION}

We have designed a tracking microwave notch filter at X-band $(8 \mathrm{GHz}-12.4 \mathrm{GHz})$ by using a pair of Gunn oscillators. The notch frequency measured in the experiment to be $9.42 \mathrm{GHz}$ and the 3$\mathrm{dB}$ bandwidth measured experimentally is $105 \mathrm{MHz}$. The theoretical response of the notch filter fits well with the experimental response. Two Gunn oscillators are injection-locked to the input

signal with the injection signal for Gunn oscillator \#2 phase shifted by $\frac{\pi}{2}$ radian at notch frequency. If the interference frequency, to be eliminated, shifts with time the notch frequency also follows the interfering signal. This is the tracking behaviour of this notch filter. The tracking range is limited within the lockband of the Gunn oscillators. The injection locking technique also reduces amplitude noise of the desired signal due to its amplitude limiting action.

\section{REFERENCES}

1. J.C. Ramadugu, "Design of microwave bandstop and bandpass filters on Barium Strontium Titanate thin film varactor technology", Ph.D. thesis, University of Dayton, Dayton, Ohio, USA, Dec. 2013.

2. P. Katzin, B.Bedard and Y. Ayasli, "Narrow-band MMIC filters with automatic tuning and Qfactor control", Proc. of IEEE International Microwave Symposium Digest, 1993, pp. 403-406, vol.1, Atlanta, GA, USA, 14-18 June, 1993. DOI: 10.1109/MWSYM.1993.276793.

3. D.R. Jackwoski and A.C. Guyette, "Sub-octave-tunable microstrip notch filter", IEEE EMC Society Symposium on Electromagnetic Compatibility, pp. 99-102, Austin, Texas, USA, Aug. 1721, 2009.

4. D.R. Jackwoski, "Passive enhancement of resonator Q in microwave notch filters", 2004 IEEE MTT-s International Microwave Symposium Digest, pp. 1351-1318, 6-11 June, 2004.

5. R.V. Snyder, "Evalution of passive and active microwave filters", Microwave Symposium Digest, pp. 1-3, Montreal, QC, Canada, 2012.

6. R. Levy, R.V. Snyder and G. Matthaei, "Design of microwave filters", IEEE Transaction on Microwave theory and Techniques, vol. MTT-50, no. 3 pp. 783-793, Mar 2002.

7. T-Lin. Wu, "Microwave filter Design", Chap.6, Department of Electrical Engineering, National Taiwan University, John Wiley \& Sons, Inc., 2001.

8. B.Y. Kapilevich, "Variety of approaches to designing microwave active filters", 27th European Microwave Conference, Jerusalem, Israel, vol. 1,pp.397-408, 8-12 Sept. 1997.

9. C.Y. Chang and T. Itoh, "Microwaves active filters based on coupled negative resistance method", IEEE Trans. On Microwave Theory and Techniques, vol. MTT-38, no. 12, pp.1879-1884, Dec. 1990.

10. M.S. Narayana and N. Gogia, "Accurate Design of a notch filter using electromagnetic simulators", Applied microwave and Wireless, pp. 44-49, vol. 12, part 11, 2000.

11. S. K. Dawn and T. Chattopadhyay, "Unilaterally Injection-Locked Gunn Oscillator pair acting as a Microwave active Notch Filter", International Journal of Electronics and Communication Engineering \& Technology (IJECET) Volume 7, Issue 2, pp. 25-32, March-April 2016.

12. B. Vidal, M.A. Piqueras, J. Marti, "Tunable and reconfigurable photonic microwave filter based on stimulated Brillouin scattering", Optics Letters, vol. 32, pp. 23-25, 2007. DOI: 10.1364/OL.32.000023

13. W. Zhang, R.A. Minasian, "Widely Tunable Single-Passband Microwave Photonic Filter Based on Stimulated Brillouin Scattering”, IEEE Photonics Technology Letters vol. 23, pp. 1775-1777, Dec. $1,2011$.

14. W. Zhang, R.A. Minasian, "Ultrawide Tunable Microwave Photonic Notch Filter Based on Stimulated Brillouin Scattering”, IEEE Photonics Technology Letters, vol. 24, pp. 1182-1184, 2012. DOI: 10.1109/LPT.2012.2198638

15. J. Sancho, N. Primerov, S. Chin, A. Zadok, S. Sale, L. Thévenaz, "Tunable and reconfigurable multi-tap microwave photonic filter based on dynamic Brillouin gratings in fibers", Optics Express, vol. 20, pp. 6157-6162, 2012. DOI: 10.1364/OE.20.006157 
16. S. Chattopadhyay and T. Chattopadhyay, "A delay line notch filter that suppresses interference", IETEJ. Research, vol. 51, No. - 4, pp. 273-277, Jul-Aug 2005.

17. T. Chattopadhyay and S. Chattopadhyay, "A microwave Notch filter", Proc. of National Conference on Radio Science (INCURSI-2003) National Physical Laboratory, New Delhi, India, Nov. 27-29, 2003.

18. T. Chattopadhyay, P. Bhattacharya, B.J. Mondal and S.D. Mazumder, "Design and analysis of a microwave notch filter using a pair of magic tees", Proc. of International Conference on Microwaves and Photonics, (ICMAP-2013), Indian School of Mines, Dhanbad, India, Dec. 13-15, 2013.

19. T. Chattopadhyay and P. Bhattacharya, "Frequency response of a Microwave notch filter in presence of two interfering tones", Proc. of the IEEE Students Tech. Symposium, pp. 347-349, IIT Kharagpur, India, 28 February-2 March, 2014.

20. I. C. Hunter and J.D. Rhodes, "Electronically tunable microwave band stop filters", IEEE transactions on microwave theory and Techniques, vol. MTT-30, pp. 1361-1367, Sept. 1982.

21. T. Chattopadhyay \& P. Bhattacharya, "Effect of Co-Channel monotone interference on the response of a microwave band reject filter", Proc. of International Microwave and RF Conference, IMaRC-2014, pp. 297-300, 15-17 December, 2014, Bangalore, India. DOI: 10.1109/IMaRC.2014.7039038.

22. T. Chattopadhyay, P.B., S.D. Mazumder and B.J. Mondal, "Design and analysis of a microwave band reject filter using a double magic tee", Proc. of International Conference on Signal Processing and Communication, 2014, (SPCOM-2014), Indian Institute of Science, Bangalore, India, 22-25 July, 2014.

23. P. Bhattacharya, S.K. Dawn \& T. Chattopadhyay, "Performance of a band reject tunable microwave filter in the face of frequency-modulated interference", Proc. of the IEEE Bombay Section Symposium, (IBSS-2015), pp.1-6, 10-11 Sept. 2015, UMIT, SNDT, Mumbai, India. DOI: 10.1109/IBSS.2015.7456651.

24. T. Chattopadhyay and S. Chattopasdhyay, "A simple tunable microwave band stop filter using two waveguide lines", Proc. of the 16th Asia Pacific Microwave Conference, (APMC-2004), University of Delhi, India, Dec. 15-18, 2004.

\section{BIBLIOGRAPHIES}

Santosh Kumar Dawn is presently pursuing his Ph.D. in Microwave and Optoelectronics Lab, Department of Physics, Visva-Bharati University. He received his M.Sc. degree from Visva-Bharati University. His topic of research is design and analysis of Microwave Filters.

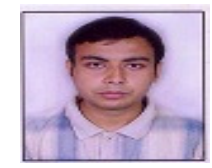

Taraprasad Chattopadhyay is a professor of Physics in Visva-Bharati (Central University) since 2003. He was awarded Japanese Govt. Scholarship by the Ministry of Education and Culture, Japan. He was an invited researcher of the Research Development Corporation, Japan. He was a fellow of AIEJ, Japan. He was a visiting professor in RIEC, Tohoku University, Japan and in AIT, Bangkok. He has authored two text books on electronics. He has about 120 publications in national and international journals and conference proceedings. He is a reviewer of many reputed international

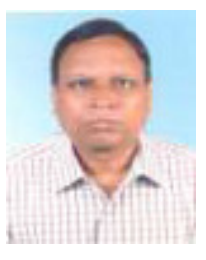
journals. He has guided and has been guiding Ph.D. students as a sole supervisor. He is a senior member of IEEE(USA) and a fellow of IETE (India).

\section{List of figures}

Fig. 1. Schematic diagram of the active, tracking microwave notch filter.

Fig. 2. Variation of output phase of Gun oscillator \#2 with injection frequency.

Fig. 3.Variation of output phase of Gun oscillator \#3 with normalized frequency detuning.

Fig. 4.Theoretical and experimental frequency response of the proposed notch filter. 